\title{
Rotational Diffusion in a Chain of Particles
}

\author{
Holger Stark $\dagger$, Michael Reichert $\dagger$, and Jérome Bibette $\ddagger$ \\ $\dagger$ Universität Konstanz, Fachbereich Physik, D-78457 Konstanz, Germany \\ $\ddagger$ Laboratoire Colloides et Matériaux Divisés, UMR 7612, ESPCI, 10 rue Vauquelin, 75005 \\ Paris, France \\ E-mail: Holger.Stark@uni-konstanz.de
}

\begin{abstract}
We study the coupled rotational diffusion in a two-particle chain on the basis of a Smoluchowski equation and calculate time-correlation functions that are measurable in an experiment. This might be used to explore hydrodynamic interactions in the limit where lubrication theory is valid.
\end{abstract}

PACS numbers: 05.45.Xt, 47.15.Gf, 47.85.Np, 82.70.Dd, 82.60.Yz

\section{Introduction}

Colloids are widely considered as models for atomic systems [1, 2]. However, a specific signature of colloidal suspensions are hydrodynamic interactions [3, 4]. Moving colloids interact with each other through the flow fields they create. This is a true multi-body problem which can only be handled by approximate methods such as multipole expansions for large particle distances (see, e.g., Refs. [4, 5]) and lubrication theory when they come close to each other [6]. Conventionally, hydrodynamic interactions are monitored through their effect on self and collective diffusion in colloidal suspensions [1 4, 7] but recent experiments with optical tweezers on a pair of particles [8] also allow a controlled exploration of hydrodynamic interactions as a function of particle separation confirming standard approaches due to Oseen and Rotne-Prager [3, 4].

Recent work also studied the rotational diffusion of tracer particles [9] 10 11] or colloids trapped in optical tweezers [12]. An experimental system introduced by Bibette et al. [13] suggests a possibility to directly measure the effect of hydrodynamic interactions on the rotational diffusion in the limit where lubrication theory is valid. Charged superparamagnetic particles under the influence of a magnetic field form chains where the particle separation can precisely be tuned by the magnetic field strength. By labeling the particles with a phosphorescent dye [11] or by using birefringent colloids [14], rotational diffusion can then be monitored.

The present article investigates rotational diffusion in a two-particle chain theoretically. It first introduces the Smoluchowski equation to treat rotational diffusion and then discusses observabels to be measured in an experiment.

\section{Rotational Diffusion and the Smoluchowski Equation}

Let us first shortly review the rotational diffusion of one particle that can also be considered as a random walk on the unit sphere. The probability density $P(\hat{\boldsymbol{\nu}}, t)$ of finding the particle 
with an orientation given by the unit vector $\hat{\boldsymbol{\nu}}$ at time $t$ satisfies the Smoluchowski equation [9]

$$
\frac{\partial P(\hat{\boldsymbol{\nu}}, t)}{\partial t}=D_{0} \nabla_{r}^{2} P(\hat{\boldsymbol{\nu}}, t) .
$$

This is in complete analogy with translational diffusion. However, instead of the Laplace operator, the square of the nabla operator in angular space $\nabla_{r}$ has to be used, where the index $r$ means rotation. Since $\boldsymbol{\nabla}_{r}=\frac{2 \pi i}{h} \boldsymbol{L}$, where $\boldsymbol{L}$ is the angular momentum operator known from quantum mechanics, all the algebra developed for $\boldsymbol{L}[15]$ is also valid for $\boldsymbol{\nabla}_{r}$. To be concrete, we note that

$$
\boldsymbol{\nabla}_{r}=\hat{\boldsymbol{\nu}} \times \frac{\partial}{\partial \hat{\boldsymbol{\nu}}}=\left(\begin{array}{c}
-\sin \varphi \frac{\partial}{\partial \vartheta}-\frac{\cos \varphi}{\tan \vartheta} \frac{\partial}{\partial \varphi} \\
-\cos \varphi \frac{\partial}{\partial \vartheta}-\frac{\sin \varphi}{\tan \vartheta} \frac{\partial}{\partial \varphi} \\
\frac{\partial}{\partial \varphi}
\end{array}\right),
$$

where $\phi, \vartheta$ are the spherical coordinates to represent $\hat{\boldsymbol{\nu}}$. The rotational diffusion constant $D_{0}$ in Eq. (1) is related via an Einstein relation to the mobility $\mu_{0}=1 /\left(8 \pi \eta a^{3}\right)$ for rotational motion, i.e., $D_{0}=k_{B} T \mu_{0}$. Now, since the operator $\nabla_{r}^{2}$ possesses the spherical harmonics $Y_{m}^{l}(\hat{\boldsymbol{\nu}})$ as its eigenfunctions, $\boldsymbol{\nabla}_{r}^{2} Y_{m}^{l}(\hat{\boldsymbol{\nu}})=-l(l+1) Y_{m}^{l}(\hat{\boldsymbol{\nu}})$, one calculates, with the same method as presented below, the time-correlation functions

$$
\left\langle Y_{m}^{l *}[\hat{\boldsymbol{\nu}}(t)] Y_{m^{\prime}}^{l^{\prime}}[\hat{\boldsymbol{\nu}}(0)]\right\rangle=\frac{1}{4 \pi} \delta_{l l^{\prime}} \delta_{m m^{\prime}} e^{-l(l+1) D_{0} t} .
$$

Here $\hat{\boldsymbol{\nu}}(t)$ means orientation of the particle at time $t$ and the symbol $*$ means complex conjugate. For small times, one shows with the help of Eq. (3) (for details, see below) that the square of the angular displacement of $\hat{\boldsymbol{\nu}}$ exhibits the typical diffusive behavior:

$$
\left\langle|\hat{\boldsymbol{\nu}}(t)-\hat{\boldsymbol{\nu}}(0)|^{2}\right\rangle \approx 4 D_{0} t .
$$

We now concentrate on a chain of two particles of radius $a$ whose centers are connected by the vector $\boldsymbol{r}$. For simplicity, we only consider their rotational diffusion, i.e., we disregard any coupling to translational motion. We especially take the particles' separation $r$ as fixed, e.g., by assuming that fluctuations around the equilibrium separation, governed by the twoparticle potential, are negligibly small. The coupled rotational diffusion is described by selfdiffusion tensors $\boldsymbol{D}_{11}=\boldsymbol{D}_{22}$ for particle 1 and 2, respectively, and the tensors $\boldsymbol{D}_{12}=\boldsymbol{D}_{21}$ encoding the hydrodynamic interactions between particle 1 and 2. As in the one-particle case, these quantities are related to mobilities by an Einstein relation, $\boldsymbol{D}_{i j}=k_{B} T \boldsymbol{\mu}_{i j}$. The mobilites $\boldsymbol{\mu}_{i j}$ connect the torque $\boldsymbol{T}_{j}$ on particle $j$ with the angular velocity $\boldsymbol{\omega}_{i}$ of particle $i: \boldsymbol{\omega}_{i}=\boldsymbol{\mu}_{i j} \boldsymbol{T}_{j}$. Due to Lorentz's reciprocal theorem [3], they fulfill $\boldsymbol{\mu}_{i j}=\boldsymbol{\mu}_{j i}^{t}$, where $t$ means transposed matrix. If, in addition, the particles are identical, $\boldsymbol{\mu}_{i j}=\boldsymbol{\mu}_{j i}$. The uniaxial symmetry of the two-particle chain determines the form of $\boldsymbol{D}_{i j}$ :

$$
\boldsymbol{D}_{i j}=D_{i j}^{\perp} \mathbf{1}+\Delta D_{i j} \hat{\boldsymbol{r}} \otimes \hat{\boldsymbol{r}} \text { with } \Delta D_{i j}=D_{i j}^{\|}-D_{i j}^{\perp},
$$

where 1 means unit tensor, $\hat{\boldsymbol{r}}=\boldsymbol{r} / r$, and $\otimes$ means tensor product. The constants $D_{i j}^{\|}$and $D_{i j}^{\perp}$ refer, respectively, to rotational diffusion about the two-particle axis $\hat{\boldsymbol{r}}$ or a direction perpendicular to it. The related mobilities as a function of reduced particle distance $r / a$ are plotted in Fig. 1] The library HydroLib [16] allows to calculate their values ranging from small particle distances where lubrication theory has to be applied to large distances where expansions into $a / r$ are applicable. For comparison, the Rotne-Prager approximation is also shown. Both graphs of Fig. 1 illustrate that the Rotne-Prager approximation works well for center-to-center distances larger than $3 a$. To see noticable deviations of the mobilities $\mu_{11}^{r r \|}$ and $\mu_{11}^{r r \perp}$ from the single-particle value $\mu_{0}=1 /\left(8 \pi \eta a^{3}\right)$ (the index $r r$ refers directly to 

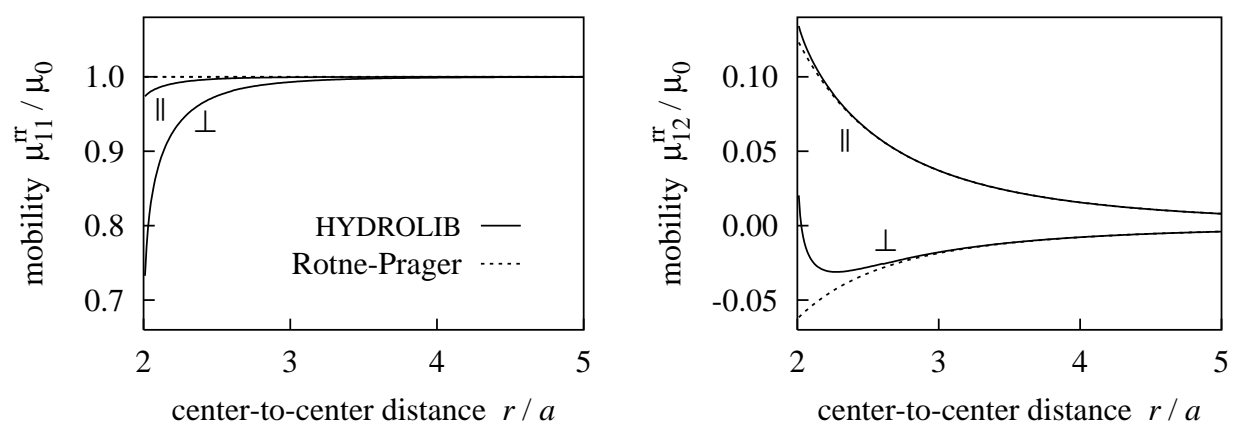

Figure 1. The rotational mobilities of the two-particle chain in units of $\mu_{0}=1 /\left(8 \pi \eta a^{3}\right)$ as a function of reduced center-to-center distance $r / a$. Full lines: values calculated with numerical library HydroLib; dotted lines: the Rotne-Prager approximation.

the rotational degree of freedom), the particles have to be close. Note that the mobilities in both graphs stay finite when the particles approach contact at $r=2 a$; the derivatives of the perpendicular coefficients, however, are singular.

The Smoluchowski equation determines the temporal evolution of the probability density $P\left(\hat{\boldsymbol{\nu}}_{1}, \hat{\boldsymbol{\nu}}_{2}, t\right)$ to find particles 1 and 2 with respective orientations given by $\hat{\boldsymbol{\nu}}_{1}$ and $\hat{\boldsymbol{\nu}}_{2}$ at time $t$ [4],9]:

$$
\frac{\partial P\left(\hat{\boldsymbol{\nu}}_{1}, \hat{\boldsymbol{\nu}}_{2}, t\right)}{\partial t}=\hat{\boldsymbol{L}}_{S} P\left(\hat{\boldsymbol{\nu}}_{1}, \hat{\boldsymbol{\nu}}_{2}, t\right)
$$

where

$$
\hat{\boldsymbol{L}}_{S}=\boldsymbol{\nabla}_{r 1} \cdot \boldsymbol{D}_{11} \boldsymbol{\nabla}_{r 1}+\boldsymbol{\nabla}_{r 2} \cdot \boldsymbol{D}_{22} \boldsymbol{\nabla}_{r 2}+2 \boldsymbol{\nabla}_{r 1} \cdot \boldsymbol{D}_{12} \boldsymbol{\nabla}_{r 2}
$$

denotes the Smoluchowski operator that is a generalization of $D_{0} \nabla_{r}^{2}$ in the single-particle equation (11. Since both particles are identical and with the help of Eq. (5), it is rewritten as

$$
\begin{aligned}
\hat{\boldsymbol{L}}_{S}= & D_{11}^{\perp}\left(\boldsymbol{\nabla}_{r 1}^{2}+\nabla_{r 2}^{2}\right)+\Delta D_{11}\left(\frac{\partial^{2}}{\partial \varphi_{1}^{2}}+\frac{\partial^{2}}{\partial \varphi_{2}^{2}}\right) \\
& +2 D_{12}^{\perp} \nabla_{r 1} \cdot \nabla_{r 2}+2 \Delta D_{12} \frac{\partial}{\partial \varphi_{1}} \frac{\partial}{\partial \varphi_{2}}
\end{aligned}
$$

where $\partial / \partial \varphi_{i}$ is the $z$ component of $\boldsymbol{\nabla}_{r i}$ with $\hat{\boldsymbol{z}} \| \boldsymbol{r}$. In analogy to the wave function determined from Schrödinger's equation in quantum mechanics, the time evolution of the probability density $P\left(\hat{\boldsymbol{\nu}}_{1}, \hat{\boldsymbol{\nu}}_{2}, t\right)$ is known in principle when eigenvalues and eigenfunctions of the Smoluchowski operator are known. Due to the analogy with the angular-momentum algebra, the eigenvectors of the unperturbed problem, i.e., particles not coupled by hydrodynamic interactions $\left(D_{12}^{\perp}=\Delta D_{12}=0\right)$, are just products of two spherical harmonics: $\Phi\left(\hat{\boldsymbol{\nu}}_{1}, \hat{\boldsymbol{\nu}}_{2}\right)=$ $Y_{m_{1}}^{l_{1}}\left(\hat{\boldsymbol{\nu}}_{1}\right) Y_{m_{2}}^{l_{1}}\left(\hat{\boldsymbol{\nu}}_{2}\right)$. They are even eigenfunctions of the last term in the second line of Eq. 8 . The operator $\nabla_{r 1} \cdot \nabla_{r 2}$, however, mixes the eigenfunctions of the unperturbed problem. How this is done, can be calculated with the help of "ladder operators" introduced in full analogy to the angular momentum algebra [15]:

$$
\nabla_{r}^{ \pm}=\nabla_{r x} \pm i \nabla_{r y}
$$

Applied to spherical harmonics, they yield

$$
\nabla_{r}^{ \pm} Y_{m}^{l}(\hat{\boldsymbol{\nu}})=i \sqrt{l(l+1)-m(m \pm 1)} Y_{m \pm 1}^{l}(\hat{\boldsymbol{\nu}})=i c_{l m}^{ \pm} Y_{m \pm 1}^{l}(\hat{\boldsymbol{\nu}})
$$


We rewrite the crucial term $\nabla_{r 1} \cdot \nabla_{r 2}$ of $\hat{\boldsymbol{L}}_{S}$ with the help of

$$
\nabla_{r x}=\frac{1}{2}\left(\nabla_{r}^{+}+\nabla_{r}^{-}\right) \text {and } \nabla_{r y}=\frac{1}{2 i}\left(\nabla_{r}^{+}-\nabla_{r}^{-}\right)
$$

as

$$
\nabla_{r 1} \cdot \nabla_{r 2}=\frac{1}{2}\left(\nabla_{r 1}^{+} \nabla_{r 2}^{-}+\nabla_{r 1}^{-} \nabla_{r 2}^{+}\right)+\frac{\partial}{\partial \varphi_{1}} \frac{\partial}{\partial \varphi_{2}}
$$

and can now fully determine how the Smoluchowski operator acts on the unperturbed eigenfunction:

$$
\begin{aligned}
-\hat{\boldsymbol{L}}_{S} Y_{m_{1}}^{l_{1}} Y_{m_{2}}^{l_{2}}= & \left\{D_{11}^{\perp}\left[l_{1}\left(l_{1}+1\right)+l_{2}\left(l_{2}+1\right)\right]+\Delta D_{11}\left(m_{1}^{2}+m_{2}^{2}\right)+2 D_{12}^{\|} m_{1} m_{2}\right\} Y_{m_{1}}^{l_{1}} Y_{m_{2}}^{l_{2}} \\
& +D_{12}^{\perp}\left(c_{l_{1} m_{1}}^{+} c_{l_{2} m_{2}}^{-} Y_{m_{1}+1}^{l_{1}} Y_{m_{2}-1}^{l_{2}}+c_{l_{1} m_{1}}^{-} c_{l_{2} m_{2}}^{+} Y_{m_{1}-1}^{l_{1}} Y_{m_{2}+1}^{l_{2}}\right)
\end{aligned}
$$

where we used $D_{12}^{\|}=D_{12}^{\perp}+\Delta D_{12}$ and the coefficients $c_{l m}^{ \pm}$are defined in Eq. (10).

\section{Time Correlation Functions and Observables}

We are interested in quantities that can be measured in experiments. We therefore define the most general time correlation function

$$
\Gamma\left(\begin{array}{l}
l_{1} l_{2} m_{1} m_{2} \\
l_{1}^{\prime} l_{2}^{\prime} m_{1}^{\prime} m_{2}^{\prime}
\end{array} t\right)=\left\langle Y_{m_{1}}^{l_{1} *}\left[\hat{\boldsymbol{\nu}}_{1}(t)\right] Y_{m_{2}}^{l_{2} *}\left[\hat{\boldsymbol{\nu}}_{2}(t)\right] Y_{m_{1}^{\prime}}^{l_{1}^{\prime}}\left[\hat{\boldsymbol{\nu}}_{1}(0)\right] Y_{m_{2}^{\prime}}^{l_{2}^{\prime}}\left[\hat{\boldsymbol{\nu}}_{2}(0)\right]\right\rangle
$$

which can be related to useful observables. Formally, $\Gamma$ is calculated using the propagator $P\left(\hat{\boldsymbol{\nu}}_{1}, \hat{\boldsymbol{\nu}}_{2}, t \mid \hat{\boldsymbol{\nu}}_{1}^{\prime}, \hat{\boldsymbol{\nu}}_{2}^{\prime}, 0\right)$ that gives the probability of finding the two particles with orientations $\hat{\boldsymbol{\nu}}_{1}$ and $\hat{\boldsymbol{\nu}}_{2}$ at time $t$ when they had with certainty the orientations $\hat{\boldsymbol{\nu}}_{1}^{\prime}$ and $\hat{\boldsymbol{\nu}}_{2}^{\prime}$ at time $t=0$ :

$$
\begin{aligned}
\Gamma\left(\begin{array}{l}
l_{1} l_{2} m_{1} m_{2} \\
l_{1}^{\prime} l_{2}^{\prime} m_{1}^{\prime} m_{2}^{\prime}
\end{array}\right)= & \iint Y_{m_{1}}^{l_{1} *}\left(\hat{\boldsymbol{\nu}}_{1}\right) Y_{m_{2}}^{l_{2} *}\left(\hat{\boldsymbol{\nu}}_{2}\right) P\left(\hat{\boldsymbol{\nu}}_{1}, \hat{\boldsymbol{\nu}}_{2}, t \mid \hat{\boldsymbol{\nu}}_{1}^{\prime}, \hat{\boldsymbol{\nu}}_{2}^{\prime}, 0\right) \\
& \times W\left(\hat{\boldsymbol{\nu}}_{1}^{\prime}, \hat{\boldsymbol{\nu}}_{2}^{\prime}, t=0\right) Y_{m_{1}^{\prime}}^{l_{1}^{\prime}}\left(\hat{\boldsymbol{\nu}}_{1}^{\prime}\right) Y_{m_{2}^{\prime}}^{l_{2}^{\prime}}\left(\hat{\boldsymbol{\nu}}_{2}^{\prime}\right) d \hat{\boldsymbol{\nu}}_{1} d \hat{\boldsymbol{\nu}}_{2} d \hat{\boldsymbol{\nu}}_{1}^{\prime} d \hat{\boldsymbol{\nu}}_{2}^{\prime}
\end{aligned}
$$

where $W\left(\hat{\boldsymbol{\nu}}_{1}^{\prime}, \hat{\boldsymbol{\nu}}_{2}^{\prime}, t=0\right)$ is the probability distribution for $\hat{\boldsymbol{\nu}}_{1}^{\prime}$ and $\hat{\boldsymbol{\nu}}_{2}^{\prime}$ at $t=0$. In the following, we will use an isotropic distribution $W=1 /(4 \pi)^{2}$ since the interaction potential of the particles does not depend on their orientations. However, one could also think about a situation where one first "aligns" the particles and then lets them evolve with time $t$. In such a case, $W$ would be given by a product of delta functions. The time evolution of the correlation function is calculated from a master equation that we derive by taking the time derivative of Eq. (15), then using the Smoluchowski equation (6) for the propagator and finally letting $\hat{\boldsymbol{L}}_{S}=\hat{\boldsymbol{L}}_{S}^{+}$act on the spherical harmonics at time $t$ :

$$
\begin{aligned}
\frac{\partial}{\partial t} \Gamma\left(\begin{array}{l}
l_{1} l_{2} m_{1} m_{2} \\
l_{1}^{\prime} l_{2}^{\prime} m_{1}^{\prime} m_{2}^{\prime}
\end{array} \mid t\right)=\iint\left[\hat{\boldsymbol{L}}_{S} Y_{m_{1}}^{l_{1} *}\left(\hat{\boldsymbol{\nu}}_{1}\right) Y_{m_{2}}^{l_{2} *}\left(\hat{\boldsymbol{\nu}}_{2}\right)\right] P\left(\hat{\boldsymbol{\nu}}_{1}, \hat{\boldsymbol{\nu}}_{2}, t \mid \hat{\boldsymbol{\nu}}_{1}^{\prime}, \hat{\boldsymbol{\nu}}_{2}^{\prime}, 0\right) \\
\quad \times W\left(\hat{\boldsymbol{\nu}}_{1}^{\prime}, \hat{\boldsymbol{\nu}}_{2}^{\prime}, t=0\right) Y_{m_{1}^{\prime}}^{l_{1}^{\prime}}\left(\hat{\boldsymbol{\nu}}_{1}^{\prime}\right) Y_{m_{2}^{\prime}}^{l_{2}^{\prime}}\left(\hat{\boldsymbol{\nu}}_{2}^{\prime}\right) d \hat{\boldsymbol{\nu}}_{1} d \hat{\boldsymbol{\nu}}_{2} d \hat{\boldsymbol{\nu}}_{1}^{\prime} d \hat{\boldsymbol{\nu}}_{2}^{\prime} .
\end{aligned}
$$

With the help of Eq. (13) and the defintion (15), the master equation assumes the form

$$
\begin{aligned}
& -\frac{\partial}{\partial t} \Gamma\left(\begin{array}{l}
l_{1} l_{2} m_{1} m_{2} \\
l_{1}^{\prime} l_{2}^{\prime} m_{1}^{\prime} m_{2}^{\prime}
\end{array} t\right)=\left\{D_{11}^{\perp}\left[l_{1}\left(l_{1}+1\right)+l_{2}\left(l_{2}+1\right)\right]+\Delta D_{11}\left(m_{1}^{2}+m_{2}^{2}\right)\right. \\
& \left.+2 D_{12}^{\|} m_{1} m_{2}\right\} \Gamma\left(\begin{array}{l}
l_{1} l_{2} m_{1} m_{2} \\
l_{1}^{\prime} l_{2}^{\prime} m_{1}^{\prime} m_{2}^{\prime}
\end{array}\right) \\
& +D_{12}^{\perp}\left[c_{l_{1} m_{1}}^{+} c_{l_{2} m_{2}}^{-} \Gamma\left({ }_{l_{1}^{\prime} l_{2}^{\prime} m_{1}^{\prime} m_{2}^{\prime}}^{l_{1} l_{2} m_{1}+1 m_{2}-1} \mid t\right)+c_{l_{1} m_{1}}^{-} c_{l_{2} m_{2}}^{+} \Gamma\left({ }_{l_{1}^{\prime} l_{2}^{\prime} m_{1}^{\prime} m_{2}^{\prime}}^{l_{1} l_{2} m_{1}-1} \mid t\right)\right]
\end{aligned}
$$

The time evolution of various observables can now be calculated with the help of this equation. We illustrate two cases which should be measurable in experiments. 


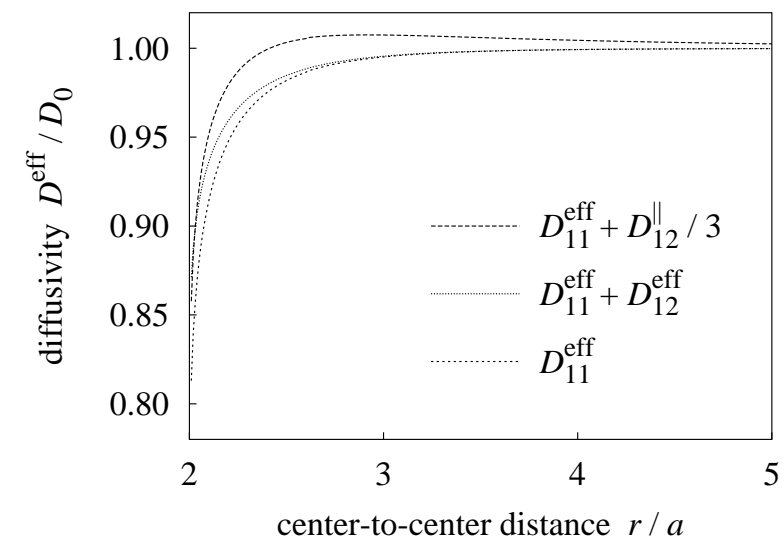

Figure 2. The effective rotational diffusion constants $D_{11}^{\text {eff }}, D_{11}^{\text {eff }}+D_{12}^{\|} / 3$, and $D_{11}^{\text {eff }}+D_{12}^{\text {eff }}$ in units of $D_{0}$ as a function of the reduced particle separation $r / a$.

\subsection{One-particle diffusion}

Let us investigate the time correlation function for the orientation of particle 1:

$$
\left\langle\hat{\boldsymbol{\nu}}_{1}(t) \cdot \hat{\boldsymbol{\nu}}_{1}(0)\right\rangle=\frac{4 \pi}{3} \sum_{m=-1}^{1}\left\langle Y_{m}^{1 *}\left[\hat{\boldsymbol{\nu}}_{1}(t)\right] Y_{m}^{1}\left[\hat{\boldsymbol{\nu}}_{1}(0)\right]\right\rangle=\frac{(4 \pi)^{2}}{3} \Gamma\left({ }_{10 m 0}^{10 m 0} \mid t\right)(
$$

The first equality is just the addition theorem for spherical harmonics [15] and the second equality used $l_{2}=l_{2}^{\prime}=m_{2}=m_{2}^{\prime}=0$, i.e., $Y_{0}^{0}=1 / \sqrt{4 \pi}$ for the second particle in the definition (14) of $\Gamma(\ldots \mid t)$. Since $c_{00}^{+/-}=0, \Gamma\left(\begin{array}{l}10 \mathrm{~m} 0 \\ 10 \mathrm{~m} 0\end{array} \mid t\right)$ does not couple to other $\Gamma$ 's. The evolution equation is therefore simple and gives

$$
\Gamma\left(\begin{array}{l}
10 m 0 \\
10 m 0
\end{array} \mid t\right)=\Gamma\left(\begin{array}{l}
10 m 0 \\
10 m 0
\end{array} \mid 0\right) e^{-\left(2 D_{11}^{\perp}+\Delta D_{11} m^{2}\right) t}
$$

Compared to the single-particle result of Eq. (3) $(l=1)$, the decay rate of the correlation function now also depends on the azimuthal quantum number $m$ due to presence of the second particle. With $\Gamma\left(\begin{array}{c}10 m 0 \\ 10 m 0\end{array} \mid 0\right)=1 /(4 \pi)^{2}$ [isotropic distribution of $\hat{\boldsymbol{\nu}}_{1}(0)$ ], the correlation function (18) becomes

$$
\left\langle\hat{\boldsymbol{\nu}}_{1}(t) \cdot \hat{\boldsymbol{\nu}}_{1}(0)\right\rangle=\frac{1}{3} e^{-2 D_{11}^{\perp} t}\left(1+2 e^{-\Delta D_{11} t}\right) .
$$

Finally, the mean-square angular displacement of $\hat{\boldsymbol{\nu}}_{1}(t)$ is calculated from \langle|$\hat{\boldsymbol{\nu}}_{1}(t)-$ $\left.\left.\hat{\boldsymbol{\nu}}_{1}(0)\right|^{2}\right\rangle=2\left(1-\left\langle\hat{\boldsymbol{\nu}}_{1}(t) \cdot \hat{\boldsymbol{\nu}}_{1}(0)\right\rangle\right)$ and for small times it reads

$$
\left\langle\left|\hat{\boldsymbol{\nu}}_{1}(t)-\hat{\boldsymbol{\nu}}_{1}(0)\right|^{2}\right\rangle \approx 4 D_{11}^{\mathrm{eff}} t \text { with } D_{11}^{\mathrm{eff}}=\frac{2 D_{11}^{\perp}+D_{11}^{\|}}{3} .
$$

So $D_{0}$ in the analogous single-particle equation (4) is replaced by the average of the selfdiffusion constants $D_{11}^{\perp}$ and $D_{11}^{\|}$that encode the presence of the second particle. The effective diffusion constant $D_{11}^{\mathrm{eff}}$ in units of $D_{0}$ is plotted in Fig. 22 as a function of the reduced particle separation $r / a$. 


\subsection{Two-particle diffusion}

To access the diffusion constant $D_{12}^{\|}$, we consider the correlation function

$$
\left\langle\hat{\boldsymbol{\nu}}_{1}(t) \cdot \hat{\boldsymbol{\nu}}_{2}(t) \hat{\boldsymbol{\nu}}_{1}(0) \cdot \hat{\boldsymbol{\nu}}_{2}(0)\right\rangle=\left(\frac{4 \pi}{3}\right)^{2} \sum_{m_{1}=-1}^{1} \sum_{m_{2}=-1}^{1} \Gamma\left(\begin{array}{l}
11 m_{1} m_{1} \\
11 m_{2} m_{2}
\end{array}\right) .
$$

The correlation functions $\Gamma\left(\begin{array}{l}11 \pm 1 \pm 1 \\ 11 m_{2} m_{2}\end{array} \mid t\right)$ do not couple to other $\Gamma$ 's and therefore relax with a rate $2\left(D_{11}^{\perp}+D_{11}^{\|}+D_{12}^{\|}\right)$as determined from the first two lines of Eq. (17). The quantity $\Gamma\left(\begin{array}{l}1100 \\ 11 m_{2} m_{2}\end{array} \mid t\right)$ couples to $\Gamma\left(\begin{array}{l}111-1 \\ 11 m_{2} m_{2}\end{array} \mid t\right)$ and $\Gamma\left(\begin{array}{l}11-11 \\ 11 m_{2} m_{2}\end{array} \mid t\right)$ but can be determined straightforwardly from the three coupled evolution equations. So, ultimately the correlation function (22) appears as a sum of three exponentials. Since we are mainly interested in the short-time limit of Eq. (22), we present a shortcut towards the result. With the Taylor expansion

$$
\Gamma\left(\begin{array}{c}
11 m_{1} m_{1} \\
11 m_{2} m_{2}
\end{array} \mid t\right)=\frac{1}{(4 \pi)^{2}} \delta_{m_{1} m_{2}}-a_{m_{1}} t,
$$

where the coefficients $a_{m_{1}}$ are directly determined from the master equation 17 using $\Gamma\left(\begin{array}{l}111-1 \\ 11 m_{2} m_{2}\end{array} \mid 0\right)=\Gamma\left(\begin{array}{l}11-11 \\ 11 m_{2} m_{2}\end{array} \mid 0\right)=0$, we immediately arrrive at

$$
\left\langle\hat{\boldsymbol{\nu}}_{1}(t) \cdot \hat{\boldsymbol{\nu}}_{2}(t) \hat{\boldsymbol{\nu}}_{1}(0) \cdot \hat{\boldsymbol{\nu}}_{2}(0)\right\rangle \approx \frac{1}{3}\left[1-4\left(D_{11}^{\mathrm{eff}}+D_{12}^{\|} / 3\right) t\right] .
$$

Note that $D_{11}^{\text {eff }}$, familiar from the one-particle diffusion [see Eq. (21], is modified here by $D_{12}^{\|} / 3$. Figure 2 2 illustrates $D_{11}^{\text {eff }}+D_{12}^{\|} / 3$ as a function of particle separation.

In the same manner, we can also determine the short-time limit of

$$
\left\langle\hat{\boldsymbol{\nu}}_{1}(t) \cdot \hat{\boldsymbol{\nu}}_{2}(0) \hat{\boldsymbol{\nu}}_{2}(t) \cdot \hat{\boldsymbol{\nu}}_{1}(0)\right\rangle=\left(\frac{4 \pi}{3}\right)^{2} \sum_{m_{1}=-1}^{1} \sum_{m_{2}=-1}^{1} \Gamma\left(\begin{array}{l}
11 m_{1} m_{2} \\
11 m_{2} m_{1}
\end{array} \mid t\right)
$$

and obtain

$$
\left\langle\hat{\boldsymbol{\nu}}_{1}(t) \cdot \hat{\boldsymbol{\nu}}_{2}(0) \hat{\boldsymbol{\nu}}_{2}(t) \cdot \hat{\boldsymbol{\nu}}_{1}(0)\right\rangle \approx \frac{1}{3}\left[1-4\left(D_{11}^{\mathrm{eff}}+D_{12}^{\mathrm{eff}}\right) t\right]
$$

with

$$
D_{12}^{\mathrm{eff}}=\frac{2 D_{12}^{\perp}+D_{12}^{\|}}{3} .
$$

We again plot $D_{11}^{\text {eff }}+D_{12}^{\text {eff }}$ in Fig. 2] A comparison of all three effective diffusion constants in Fig. 2. only reveals small differences that pose a challenge for measurements in an experiment. Nevertheless, for small particle distances, the effect of hydrodynamic interactions calculated on the basis of lubrication theory should be measurable.

A natural extension of the theory presented here is a chain of more than two particles. However, the mobilites or diffusion tensors in such a system cannot any longer be described by the two-particle system; when a particle is situated between two other colloids, threebody effects become important, certainly for small separations. So experiments could be used to measure deviations from the effective diffusion constants presented in this article. Furthermore, the coupling to positional fluctuations of the particles has to be incorporated. 


\section{References}

[1] Pusey P N 1991 in Liquids, Freezing, and Glass Transition, Proceedings of the Les Houches Summer School of Theoretical Physics 1989, Part II, ed J P Hansen, D Levesque, and J Zinn-Justin (Amsterdam: NorthHolland), p 763

[2] Poon W, Pusey P, and Lekkerkerker H April 1996 Physics World 27

[3] Happel J and Brenner H 1973 Low Reynolds number hydrodynamics (Leyden: Noordhoff)

[4] Dhont J K G 1996 An Introduction to Dynamics of Colloids (Amsterdam: Elsevier)

[5] Cichocki B, Felderhof B U, Hinsen K, Wajnryb E, and Bławzdziewicz J 1994 J. Chem. Phys. 1003780

[6] Kim S and Karrila S J 1991 Microhydrodynamics: Principles and Selected Applications (Boston: ButterworthHeinemann)

[7] Nägele G 1996 Phys. Rep. 272215

[8] Meiners J-C and Quake S R 1999 Phys. Rev. Lett. 82 2211; Bartlett P, Henderson S I, and Mitchell S J 2001 Philos. Trans. R. Soc. London A 359 883; Henderson S, Mitchell S, and Bartlett P 2001 Phys. Rev. E 64 061403

[9] Montgomery J A and Berne B J 1977 J. Chem. Phys. 104589

[10] Jones R B 1988 Physica A 150 339; Jones R B 1989 Physica A 157 752; Degiorgio V, Piazza R, and Jones R B 1995 Phys. Rev. E 522707

[11] Koenderink G, Zhang H, Lettinga M P, Nägele G, and Philipse A P 2001 Phys. Rev. E 64022401

[12] Reichert M and Stark H 2004 Phys. Rev. E 69031407

[13] Goubault C, et al. 2003 Phys. Rev. Lett. 91 260802; Cohen-Tannoudji L, et al. 2005 Phys. Rev. Lett. 94038301

[14] Mertelj A, Arauz Lara J L, Maret G, Gisler T, and Stark H 2002 Europhys. Lett. 59337

[15] Messiah A 1961 Quantum Mechanics, Vol. I an II (Amsterdam: North-Holland)

[16] Hinsen K 1995 Comp. Phys. Comm. 88327 